\title{
Mathematical model of orientation of a ferromagnetic particle in magnetic field
}

\author{
Eugene Masyutkin ${ }^{1, *}$, Vasilii Masyagin ${ }^{2}$, and Boris Avdeyev ${ }^{1}$ \\ ${ }^{1}$ Kerch State Maritime Technological University, Ordzhonikidze st., 82, Kerch, 298309, Russia \\ ${ }^{2}$ Omsk State Technical University, Mira 11, Omsk, 644050, Russia.
}

\begin{abstract}
A mathematical model of the rotational motion of a particle with pronounced ferromagnetic properties, due to the moment of forces from the side of an external homogeneous magnetic field is deduced in the article. The basis of the model is the equation for the moment of forces effecting on a solid body. The driving torque is the magnetic moment, calculated through the strength of the external field and the magnetization of the material. The counter-torque is the moment of medium resistance, which mainly depends on the viscosity of the medium in which the simulated body is located. The dependences of the angle of rotation and angular velocity on time are determined. The mathematical model is represented as a system of differential equations. The developed mathematical model was solved by a Runge - Kutta fourth order method. The obtained results are presented in the form of graphs.
\end{abstract}

\section{A problem statement}

The use of magnetic properties of materials has found wide application not only in electrical engineering, but also in machine building and mining and processing complex. As well-known examples of the use of devices based on permanent magnets or electromagnets, it is possible to bring electromagnets for moving massive metal objects, magnetic plates similar in operating principle to the function of the vice grip, magnetic and electromagnetic couplings, magnetic bearings, solenoid valves and cranes of the linear and rotary drive, magnetostrictive devices, separation of ferromagnetic impurities from cooling lubricants, removing of cutting or grinding products in metal processing, etc. [1-3].

One should include a huge number of various displacement sensors, torsion sensors, angle of rotation sensors, limit switches sensors, level gauges, based on the change in the force of attraction / rotation of the magnet or electrical resistance under external influence in a special group. The main advantage of using magnetic forces is a good fixation index, as well as relatively small device sizes.

\footnotetext{
* Corresponding author: dirigeant@mail.ru
} 


\section{Objectives and tasks of work}

It becomes necessary to calculate magnetic devices such as dimensions and mass, specific gravity or distance between poles in the process of manufacturing devices using magnetic fields for their work. Calculations of the linear motion of ferromagnetic parts do not cause any special difficulties because of the topic is investigated, the calculation process of rotational motion are complex. The orientation of particles or parts of mechanisms under the action of a magnetic field is especially insufficiently explored.

The work objective is to describe model of the rotational motion of a spherical particle by strongly pronounced magnetic properties caused by the moment of forces from external uniform magnetic field.

\section{The main part}

As a rule, the motion of a particle in the viscous medium is described in the assumption of proportionality of resistance force affecting on a particle, its speeds. Such classical consideration of the motion allows getting the differential equation of the motion by which it is possible to define any statistical characteristics [4,5].

Let's write down rotation of the magnetic particle placed in constant magnetic field and caused by its orientation along magnetic field line by means of the differential equation for the moment of forces affecting on a particle. The real motion of a fine-dispersed particle in the viscous medium is accompanied by increase of particles' sizes. It leads to manifestation of hereditary properties of an impulse, and, the less particle is, the more capture of particles of the viscous medium influences its orientation.

Let us assume that the magnetic particle has spherical shape. The equation for rotary motion has a form [6]:

$$
J \frac{d \omega}{d t}=M_{M a g}+M_{D}+\xi(\mathrm{t})
$$

where $\omega$ - the angular speed of particle rotation about axis, $\mathrm{s}^{-1} ; M_{M a g}-$ an external couple moment affecting on a particle, $\mathrm{N} \cdot \mathrm{m} ; M_{D}$ - the torque of drag force, $\mathrm{N} \cdot \mathrm{m} ; \xi(t)$ - the random moment of forces, $\mathrm{N} \cdot \mathrm{m}$.

The moment of inertia of a spherical particle about the axis of rotation passing through its center of mass is calculated from formula:

$$
J=\alpha \cdot m \cdot \frac{d^{2}}{4}=\frac{\pi}{60} \cdot \alpha \cdot \rho \cdot d^{5}
$$

where $\alpha \leq 1$ - coefficient, indicating irregularity of mass distribution of particle on volume (at uniform mass distribution on volume $\alpha=1$ ), dimensionles quantity; $m$ - particle mass, $\mathrm{kg} ; d$ - particle diameter, $\mathrm{m} ; \rho$ - average density of particle, $\mathrm{kg} / \mathrm{m}^{3}$.

Magnetostatic charges - magnetic poles appear on opposite surface areas of ferromagnetic particles under the action of external magnetic field of $H$. It is found experimentally that magnetic poles are characterized by "the pole size". Thus, particles become magnetic dipoles. Then a process of orientation along magnetic field lines of the external field takes place (fig. 1). 


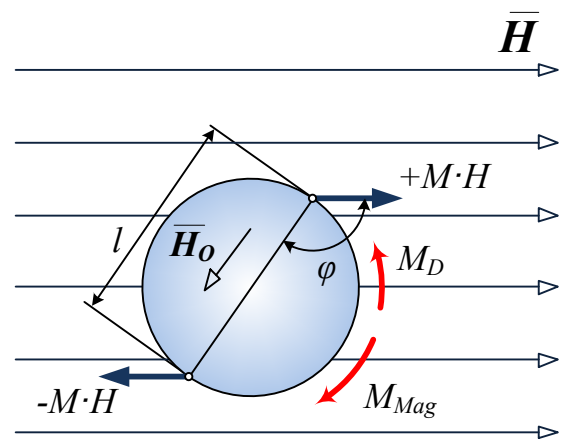

Fig. 1. Orientation of a particle under the action of magnetic field.

Forces affecting on poles are similar to forces affecting on a dipole in electric field, is calculated as the product of "the magnetic weight" and the field strength. Substituting the turning dipole in uniform magnetic field in the equation of the moment of forces, one obtains [7]:

$$
p_{M}=F \cdot l \cdot \sin (\varphi)=H \cdot M \cdot d \cdot \sin (\varphi),
$$

where $\varphi$ - the angle between the direction of the field and an axis of a dipole, rad.

The problems associated with "the magnetic masses" don't cease still. Existence of a magnetic monopole (the particle possessing a non zero magnetic charge - "the magnetic mass") is predicted by P. Dirac's hypothesis [8] though there are no experimental verifications of this hypothesis, despite of everlasting efforts of experimenters. The modern physics considers that there are no "magnetic masses". Therefore, there are no isolated magnetic poles of one sign [9]. Some researchers consider that magnetic charges should be read as current charges which are quite really [10]. Some scientists consider that the cause of arising of magnetic field is not a hypothetical particle, but the so-called "current element" equal to the product of electric current in a path and length straight run elementary path [11].

At uniform magnetization of a ferromagnetic material, its magnetic moment is connected with magnetization the $I$ following ratio:

$$
I=\frac{p_{M}}{V}
$$

Magnetization is proportional to tension of the magnetizing field [12]:

$$
I=\chi \cdot H_{I n}
$$

where $\chi$ - magnetic susceptibility of a body; $H_{I n}$ - filed strength inside the particle, $\mathrm{A} / \mathrm{m}$.

Filed strength inside the particle $H_{I n}$ is resultant of two fields - external $H$ and demagnetized one $H_{O}$ :

$$
H_{I n}=H-H_{O}
$$

$H_{O}$ is proportional to the value of magnetization and it is directed against the external field:

$$
H_{O}=N \cdot I
$$


where $N$ - demagnetization coefficient, depending mainly from geometric shape and the relative sizes of a particle and to a less extent from material.

$N$ can be precisely calculated only for ellipsoids of rotation which have uniform magnetization (for sphere $N=1 / 3$, for very thin plate $N=1$, for the infinitely long cylinder in the transverse field $N=1 / 2$ ). For some samples of an idle form $N$ is calculated from empirical formulas, but in the majority of cases of $N$ is calculated by an empirical way [13].

Filed strength inside the particle $H_{I n}$ :

$$
H_{I n}=\frac{H}{1+N \cdot \chi} .
$$

Expressing “magnetic mass" via formulae (4-8), one obtains:

$$
p_{M}=V \cdot H \cdot \frac{\chi}{1+N \cdot \chi}
$$

The moment of viscous resistance to rotation of a particle is determined by a formula [14]:

$$
M_{D}=-\pi \cdot v \cdot \rho_{f} \cdot d^{3} \cdot \omega
$$

where $v$ kinematic viscosity, Пa $\mathrm{c}$; $\rho_{f}$-density of viscous medium, $\mathrm{kg} / \mathrm{m}^{3}$.

The magnetic susceptibility of a body is calculated from the following formula

$$
\chi_{P}=\frac{\chi}{1+N \cdot \chi}
$$

Taking into account that the size of a magnetic susceptibility of ferromagnetic materials equals to about 1000 and more [15], value $N$ varies from 0,1 to 0,5 ; then one can be neglected in denominator because of its insignificance. Thus after reduction of a magnetic susceptibility of substance, it is obvious that the magnetic susceptibility of a substance depends on coefficient of demagnetization which values are influenced to a greater not by material, and by a shape of a substance as discussed earlier. Thus $\chi_{P} \sim 1 / N$ and range from 2 to 10 .

The equation of rotary motion in the absence of random action takes the following form:

$$
\left\{\begin{array}{l}
\frac{d \varphi}{d t}=\omega \\
\frac{d \omega}{d t}=\frac{10 \cdot H \cdot \chi \cdot \sin (\phi)}{\alpha \cdot \rho \cdot d^{2} \cdot(1+N \cdot \chi)}-\frac{60 \cdot v \cdot \rho_{f} \cdot \omega}{\alpha \cdot \rho \cdot d^{2}}
\end{array}\right.
$$

The system of the equations (12) was being solved with a numerical method by means of MathCAD for special cases (fig. 2): particles - iron; $d=10$ microns; medium - water; $H_{0}=4 \cdot 10^{4} \mathrm{~A} / \mathrm{m} ; \chi=10^{4} ; N=1 / 3$.

The received mathematical model can be used at further calculations of colloidal systems, the containing ferromagnetic particles.

The dipole orientation along the lines of force corresponds to the minimum energy. Let's calculate the operation done by the dipole for rotation from some angle $\varphi_{0}$ to a position which is parallel to the field. Rotation to an infinitesimal angle $\Delta \varphi$ requires an operation $\Delta \varphi p M \sin \varphi$. So, the total operation is: 


$$
\int_{\varphi 0}^{\pi} p_{M} \sin \varphi d \varphi=p_{M} \sin \varphi_{0}
$$
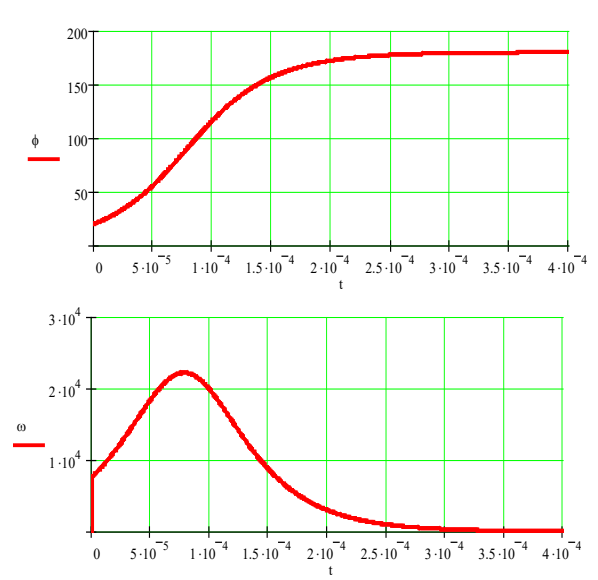

$a$
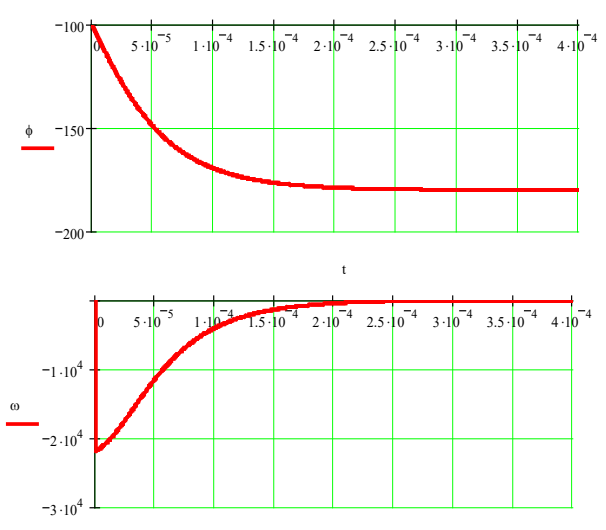

$b$

Fig. 2. Results of a numerical research of mathematical model of turn of a ferromagnetic particle in magnetic field (an angle of rotation and angular speed) at: a) $\left.\varphi_{0}=40^{\circ} ; \omega_{0}=0 \mathrm{~s}^{-1} ; b\right) \varphi_{0}=-100^{\circ} ; \omega_{0}$ $=0 \mathrm{~s}^{-1}$;

\section{Conclusion}

The considered model of rotary motion of a spherical ferromagnetic particle represents an angle of rotation and the angular speed arising at affect of magnetic field on a particle. The developed mathematical model can be used for further calculations of motion or fixation of magnetic bodies during their processing or moving. Calculations will also be useful in the modeling of colloidal systems containing ferromagnetic particles.

\section{References}

1. A.N. Shapovalov, R.R. Dema, S.P. Nefedyev, and others, J. Chem. Technol. Metall., Vol. 52 Issue 5, pp. 1008-1016 (2017)

2. V. Budnik, S. Chernyi, Procedia Eng., 150, (2016)

3. A.A. Sokolov, A.S. Miroshnikov, E.A. Sokolova, Gornyi Zhurnal, 12, 83 (2016)

4. S. Chernyi, IOP Conf. Ser.: Mater. Sci. Eng., 124 (2017)

5. B. Avdeyev, MATEC Web Conf.: Int. Conf. on Mod. Trends Manuf. Technol. Equip. 129, 06012 (2017)

6. A.N. Morozov, A.V. Skripkin, Spherical particle Brownian motion in viscous medium as non-Markovian random process, Phys. Lett. A. 375, pp. 4113-4115 (2011)

7. S.G. Kalashnikov, Electricity (Fizmatlit, Moscow, 2008)

8. P. A. M. Dirac, Proc. R. Soc. London A133, 60 (1931)

9. V.F. Mitkevich Physical fundamentals of electrical engineering (URSS, Moscow 2015)

10. Kimball A. Milton, Theoretical and experimental status of magnetic monopoles // Reports on Progress in Physics. Vol. 69. No. 6. P. 1637-1712. (2006) 
11. I.N. Aliev, I.S. Kopylov, Use of dirac monopoles formalism in some magnetism problems Herald of the Bauman Moscow State Technical University. Ser. Nat. Sc., 6 (2015)

12. S.V. Vonsovsky, Magnetism. Magnetic properties of dia, vapor, ferro, antiferro, and ferrimagnetics (Science, Moscow, 1971)

13. I.I. Kiefer, Tests of ferromagnetic materials (Energy, Moscow, 1969)

14. V.A. Zhukov, E.P. Masyutkin, B.A. Avdeyev, IOP Conf. Ser.: Mater. Sci. Eng., 177 (2017)

15. M. Sibley, Introduction to Electromagnetism (Butterworth-Heinemann, 1995) 